Pacific Journal of Mathematics

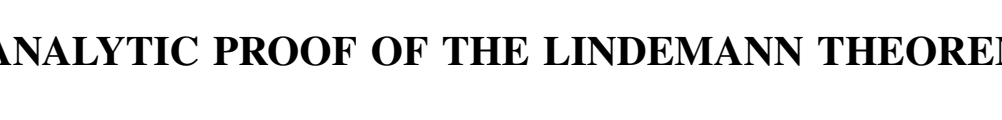




\title{
ANALYTIC PROOF OF THE LINDEMANN THEOREM
}

\author{
R. Steinberg and R. M. Redheffer
}

1. Introduction. The equation

$$
a e^{\alpha}+b e^{\beta}+\cdots+s e^{\sigma}=0
$$

would be true if all coefficients $a, b, \ldots, s$ were zero; it could be true with nonzero coefficients if any two exponents were equal; and it could be true, with nonzero coefficients and unequal exponents, if either the one or the other were transcendental. Thus $1+e^{i \pi}=0$, and $e(e)-e^{2}=0$. Now, the Lindemann theorem says that the equation can be true in these cases only:

I.INDEMANN THEOREM. The number e cannot satisfy an equation of the form (1), in which at least one coefficient $a, b, \cdots, s$ is different from zero, no two exponents $\alpha, \beta, \cdots, \sigma$ are equal, and all numbers $a, b, \cdots, s ; a, \beta, \cdots, \sigma$ are algebraic.

The proof of this goes back to Hermite [2], Lindemann [5], and Weierstrass [10]. Since then the theorem has been a favorite topic for expository articles, alternative proofs, and so on, which turn up at the rate of several per decade. One naturally asks whether any good could come of still another note on this well-worn subject.

What we have in mind here is the following. In [3] there is a discussion by Hilbert of the transcendence of $e$ and $\pi$, in which the tools are chiefly analytic in character, and in which the proof for $\pi$ is a simple extension of the proof for $e$. Hilbert remarks that the general Lindemann theorem can be obtained in the same way. This line of inquiry was followed up by Klein [4, pp. 61-77], but in the authors' opinion the treatment is not complete. What appears to be the most delicate part is relegated to a footnote. Our primary objective, then, is to supply the details in the Hermite-Hilbert proof. Naturally we have drawn heavily on [3] and [4], and the whole treatment is expository in character. The exposition is made to depend on more elementary algebraic ideas than is the case, for example, in [7, pp. 1-24] and [8, p.40].

Received August 10, 1951.

Pacific J. Math. 2 (1952), $231-242$ 
The proof divides itself naturally into two parts, analytic and algebraic. In the analytic part, a weakened form of the Lindemann theorem is proved; this is Theorem 1 of our paper. In the algebraic part, the hypotheses of the Lindemann theorem and of Theorem 1 are shown to be equivalent; the statement is given in Theorem 2.

This discussion is preceded by a proof that $e$ is transcendental, which contains the central idea of the proof of the Lindemann theorem, and yet is not beclouded by algebraic and analytic complexities. In this way the presentation is made sufficiently complete for classroom use, and at the same time the major steps in the more general theorem are well motivated. We have taken care, however, that the proof of the main theorem be logically independent of the more special discussion, and that no gaps be left for the reader to fill in by analogy.

2. Transcendence of $e$. Suppose $e$ algebraic, so that one has the special case of (1),

$$
a_{0}+a e+b e^{2}+\cdots+s e^{n}=0
$$

for integers $a_{0}, a, b, \cdots, s$ with $a_{0} \neq 0$. We try to approximate $e^{k}(k=1,2, \cdots$, $n$ ) by rational numbers $M_{k} / M$ with error small compared to the denominator $M$. Thus

$$
e^{k}=\left(M_{k}+\epsilon_{k}\right) / M
$$

where the $M$ 's are integers and the $\epsilon$ 's are small. Substituting (3) in (2) gives

$$
a_{0} M+\left(a M_{1}+b M_{2}+\cdots+s M_{n}\right)+\left(a \epsilon_{1}+b \epsilon_{2}+\cdots+s \epsilon_{n}\right)=0
$$

after multiplication by $M$. We set up the approximation (3) in such a way that the part of (4) containing $M$ 's does not vanish while the part containing $\epsilon$ 's has absolute value less than unity. The integral character of $a_{0}, a, b, \cdots$, and $M, M_{1}, M_{2}, \ldots$ shows then that (4) is impossible.

To show that the integral part of (4) does not vanish it would suffice to exhibit a prime $p>a_{0}$ that divides $M_{1}, M_{2}, \cdots, M_{n}$, but does not divide $M$. Now, an integer which has simple divisibility properties and is at the same time simply related to $e$ is given by

$$
\frac{1}{(p-1) !} \int_{0}^{\infty} e^{-z} z^{p-1} z^{k} d z= \begin{cases}\bar{m}(p) & (k=0) \\ m(p) & (k \geq 1)\end{cases}
$$

Here and elsewhere we use the notation 


$$
\begin{aligned}
& m(p)=\text { multiple of } p \\
& \bar{m}(p)=\text { integer, not multiple of } p .
\end{aligned}
$$

The truth of (5) is evident from $\Gamma(p+k)=(p+k-1)$ !, which is trivial for the integral arguments $k, p$ that occur here.

Having (5), we are ready to define the $M$ 's. The idea is to arrange matters so that the second case (5) occurs for $M_{1}, M_{2}, \ldots$ but the first occurs for $M$ itself. This is accomplished as follows. Let

$$
M=\frac{1}{(p-1) !} \int_{0}^{\infty} e^{-z} P(z) d z
$$

where

$$
P(z)=z^{p-1}[(z-1)(z-2) \cdots(z-n)]^{p} .
$$

If we imagine the bracket in $P(z)$ expanded and use (5), we see that $M=\bar{m}(p)$ whenever $p>n$. (Note, however, that if $P(z)$ had contained $z^{P}$ as a factor, then we should have obtained $m(p)$. This fact, which dictates the rather strange form (7), will be fully exploited.) From (3) we have $M_{k}+\epsilon_{k}=e^{k} M$, which suggests

$$
\begin{aligned}
M_{k} & =\frac{1}{(p-1) !} \int_{k}^{\infty} e^{k-z} P(z) d z, \\
\epsilon_{k} & =\frac{1}{(p-1) !} \int_{0}^{k} e^{k-z} P(z) d z,
\end{aligned}
$$

when we divide the range of integration at the point $k$ and put $e^{k}$ inside. The change of variable $z-k=u$ in (9) gives

$$
M_{k}=\frac{1}{(p-1) !} \int_{0}^{\infty} e^{-u} P(u+k) d u \text {. }
$$

Equation (8) shows that $P(u+k)$ contains $u^{p}$ as a factor, for $k=1,2, \cdots, n$, so that we have the second case (5); and hence $M_{k}=m(p)(k=1,2, \cdots, n)$. On the other hand for $\epsilon_{k}$ one obtains the estimate

$$
\left|\epsilon_{k}\right|<K H^{p} /(p-1) ! \quad(k=0,1, \cdots, n)
$$

by inspection of (10), and this tends to zero as $p \rightarrow \infty$. First make the prime $p>n$, so that $M=\bar{m}(p)$; then make $p>a_{0}$, so that also $a_{0} M=\bar{m}(p)$; and finally make $p$ so large that the part of (4) involving the $\epsilon$ 's is less than 1 in absolute value. 
3. Lemmas. The subsequent discussion requires certain lemmas, which we present now so that we shall not have to break the thread of the argument later. Only Lemma 1 is needed for the proof of Theorem 1 ; the others are required, however, for Theorem 2.

LEMMA 1. Let $\rho_{1}, \rho_{2}, \cdots, \rho_{m}$ be the roots of the polynomial equation $a z^{m}+b z^{m-1}+c z^{m-2}+\cdots=0$ with integral coefficients $a, b, c, \cdots$. Then any symmetric polynomial in the quantities $a \rho_{1}, a \rho_{2}, \ldots, a \rho_{m}$, with integral coefficients, is an integer.

Lemma 2. Suppose given a polynomial in $m$ variables $\alpha_{i}$, in $n$ variables $\beta_{i}, \cdots$, and in $k$ variables $\sigma_{i}$, which is symmetric in the $\alpha$ 's, in the $\beta$ 's, ..., and in the $\sigma$ 's, and which has rational coefficients. If the $\alpha$ 's are chosen to be all the roots of a polynomial equation with rational coefficients, and similarly for the $\beta$ 's, ..., and for the $\sigma$ 's, then the value of the polynomial is rational.

A polynomial is said to be irreducible over the rationals if it cannot be factored into polynomials of lower degree with rational coefficients.

LEMma 3. Any polynomial with rational coefficients can be factored into irreducible polynomials with rational coefficients.

If $\alpha_{1}$ is a root of an irreducible equation with rational coefficients, whose other roots are $\alpha_{2}, \alpha_{3}, \ldots, \alpha_{n}$, then the numbers $\alpha_{1}, \alpha_{2}, \ldots, \alpha_{n}$ are said to be the conjugates of $\alpha_{1}$. That the conjugates are well determined is shown by the following:

LEMMA 4. Over the field of rationals, an algebraic number is a root of a unique irreducible polynomial with rational coefficients and leading coefficient unity. Such an equation has no multiple roots.

LEMmA 5. The Van der Monde determinant det $\left|\left(\rho_{k}\right)^{i-1}\right|$ vanishes only if two or more of the $\rho$ 's are equal.

Lemma 1, which follows (for example) from [9, pp.63, 264], is the only one whose proof presents any difficulty. Lemma 2 follows easily from Lemma 1, by induction on the number of sets of variables; Lemmas 3 and 4 follow from the elementary theory of polynomials [1, pp.94, 95]; and Lemma 5 is a simple exercise in determinants $[9, \mathrm{p} .214$, ex. 4$]$.

Besides the algebraic lemmas given above, we shall need the following analytic ones: 
Lemma 6. Let $a, b, \cdots, s$ be any complex numbers, and let $\rho_{i}$ be any complex numbers no two of which are equal. If $\phi(z)=a z^{\rho_{1}}+b z^{\rho_{2}}+\cdots+s z^{\rho_{n}} \equiv 0$ in $z$, then $a=b=\cdots=s=0$.

To see this, let $z=1+x$ with $|x|<1$ and expand by the binomial theorem to get $\phi(1+x)$ as a convergent power series in $x$. Since the series vanishes identically by hypothesis, each coefficient does. Thus

$$
\begin{gathered}
a+b+\cdots+s=0, \\
a \rho_{1}+b \rho_{2}+\cdots+s \rho_{n}=0, \\
a \rho_{1}\left(\rho_{1}-1\right)+b \rho_{2}\left(\rho_{2}-1\right)+\cdots+s \rho_{n}\left(\rho_{n}-1\right)=0,
\end{gathered}
$$

Combining (14) with (15) changes the latter to

$$
a \rho_{1}^{2}+b \rho_{2}^{2}+\cdots+s \rho_{n}^{2}=0
$$

a similar use of (14), (16) on the next equation gives

$$
a \rho_{1}^{3}+b \rho_{1}^{3}+\cdots+s \rho_{n}^{3}=0,
$$

and so on. The lemma now follows from (13), (14), (16), (17), ..., and Lemma 5.

LEMMA 7. If a finite product of expressions like those in Lemma 6 is identically zero, then at least one expression is identically zero.

For proof, suppose that each expression has only a finite number of zeros in $1 \leq z \leq 2$. Then so does the product. At least one expression therefore has infinitely many zeros in $l \leq z \leq 2$; hence it has a limit point of zeros; and hence, by analyticity, it is identically zero.

Lemma 8. Suppose an expression $\phi(z)$ of the type considered in Lemma 6 has coefficients $a, b, \cdots, s$ which are functions of certain variables $\alpha, \beta, \cdots, \sigma$. If $\phi(z)$ is a symmetric function of $\alpha, \beta, \cdots, \sigma$ for every $z$, then the coefficients $a, b, \cdots$, are themselves symmetric functions of $\alpha, \beta, \cdots, \sigma$.

To see this, interchange two of the variables $\alpha, \beta, \cdots, \sigma$. The two functions $\dot{\phi}$ and $\phi_{1}$ after interchange are identically equal in $z$ by hypothesis, and hence corresponding coefficients are equal by Lemma 6: $a=a_{1}, b=b_{1}, \cdots, s=s_{1}$. Thus the interchange did not alter $a, b, \cdots, s$, so that these are symmetric functions by definition. 
4. Analytic part of the proof. Here we prove the following theorem, which has a generality intermediate between that of the Lindemann theorem and that of the result established in $\$ 2$ :

THEOREM 1. The number e cannot satisfy an equation of the form

$$
\begin{aligned}
a_{0} & +a\left(e^{\alpha_{1}}+e^{\alpha_{2}}+\cdots+e^{\alpha_{m}}\right) \\
& +b\left(e^{\beta_{1}}+e^{\beta_{2}}+\cdots+e^{\beta_{k}}\right) \\
& \left.\cdot \cdot \cdot \cdot \cdot \cdot \cdot \cdot \cdot \cdot e^{\sigma_{j}}\right)=0, \\
& +s\left(e^{\sigma_{1}}+e^{\sigma_{2}}+\cdots+e^{-}+\cdots\right.
\end{aligned}
$$

where $a_{0}, a, b, \ldots, s$ are nonzero integers and where the $\alpha$ 's are all of the nonzero roots of an algebraic equation with integral coefficients and lending coefficient $A$, the $\beta$ 's are the same for an equation with leading coefficient i, and so on, up to the $\sigma$ 's for which the leading coefficient is $S$.

We try first to approximate the various blocks of terms in (18) by rationial numbers, so that

$$
\begin{aligned}
& e^{\alpha_{1}}+e^{\alpha_{2}}+\cdots+e^{\alpha_{m}}=\left(M_{1}+\epsilon_{1}\right) / M, \\
& e^{\beta_{1}}+e^{\beta_{2}}+\cdots+e^{\beta_{k}}=\left(M_{2}+\epsilon_{2}\right) / M \text {, } \\
& \text {. . . . . . . . . . . } \\
& e^{\sigma_{1}}+e^{\sigma_{2}}+\cdots+e^{\sigma_{j}}=\left(M_{n}+\epsilon_{n}\right) / \|,
\end{aligned}
$$

where the M's are integers and the $\epsilon$ 's are small. Substitution of (19) into (18) gives (4) again, after multiplication by $M$. We define $\| /$ by $(7)$, where $P^{\prime}(z)$ now has the form

$$
P(z)=z^{p-1}\left[\Pi\left(z-\alpha_{i}\right) \Pi\left(z-\beta_{i}\right) \ldots \Pi\left(z-\sigma_{i}\right)\right]^{P}(A B \ldots S)^{d p}
$$

instead of the form (8), and where $d-1$ is the degree of the expression in brackets.

The hypothesis concerning the $\alpha$ 's shows that $A$ II $\left(z-\alpha_{i}\right)$ is a polynomial with integral coefficients, and similarly for $B I I\left(z-\beta_{i}\right), \ldots, S \Pi\left(z-\sigma_{i}\right)$. Ilence any product of these polynomials has integral coefficients, and therefore $P(z)$ does. The coefficient of $z^{p^{-1}}$ in $P(z)$ is 


$$
\pm\left[\left(\Pi \alpha_{i}\right)\left(\Pi \beta_{j}\right) \cdots\left(\Pi \sigma_{k}\right)\right]^{p}(A B \cdots S)^{d p}= \pm G^{p} \text {, say, }
$$

which is not zero by the hypothesis of Theorem 1. Upon substitution into (7) this term gives $\bar{m}(p)$ by (5), provided $p>G$ in (21). But the other terms in the expansion of $P(z)$ give $m(p)$ when substituted in (7), as we see again by (5); and hence $M=\bar{m}(p)$ for $p>G$.

In defining $M_{1}$ and $\epsilon_{1}$ we are guided by (9) and (10), but we take care that complete symmetry among the $\alpha$ 's be preserved; then Lemmas 1 and 8 will show (perhaps) that certain expressions which arise are integers. Let

$$
\begin{aligned}
& M_{1}=\frac{1}{(p-1) !} \sum_{i} \int_{a_{i}}^{\infty} e^{a_{i}-z} P(z) d z, \\
& \epsilon_{1}=\frac{1}{(p-1) !} \sum_{i} \int_{0}^{a_{i}} e^{a_{i}-z} P(z) d z,
\end{aligned}
$$

and similarly for $M_{2}, \epsilon_{2}, \ldots, M_{n}, \epsilon_{n}$, the role taken by the $\alpha$ 's in (22), (23) being taken respectively by the $\beta$ 's, ... or by the $\sigma$ 's. The paths of integration are, in (23), the straight lines from the origin to $\alpha_{i}$, and, in (22), the straight lines parallel to the real axis from $\alpha_{i}$ to $+\infty$. The truth of (19) is a simple consequence of Cauchy's theorem.

The substitution $z-\alpha_{i}=u$ combines with (22) to give

$$
M_{1}=\frac{1}{(p-1) !} \int_{0}^{\infty} e^{-u} \sum_{i} P\left(u+\alpha_{i}\right) d u
$$

when we interchange the order of (finite summation and integration. If we consider $P(u+w)$, with $w$ a variable, it is evident from (20) that each power of $u$ has as coefficient a polynomial in $w$ with integral coefficients. But because of the high power of $A$ that occurs as a factor of $P(z)$ in (20), these coefficients are in fact polynomials in $A w$ with integral coefficients. Setting $w=\alpha_{1}, w=$ $a_{2}, \cdots, w=\alpha_{m}$ in succession and adding, we see that $P\left(u+\alpha_{i}\right)$ has coefficients which are symmetric polynomials in $A \alpha_{1}, A \alpha_{2}, \ldots, A \alpha_{m}$ with integral coefficients. By Lemma 1 , then, they are integers.

Since $P(z)$ is divisible by $\left(z-a_{i}\right) P$, we know that $P\left(u+a_{i}\right)$ is divisible by $u^{p}$. Hence $P\left(u+\alpha_{i}\right)$ is also divisible by $u^{p}$, and the fact that the coefficients are integers combines with (24) and (5) to give $M_{1}=m(p)$. Similarly $M_{2}=$ $m(p), \cdots, M_{n}=m(p)$.

By inspection of (23) we get an estimate of the type (12); specifically, 


$$
(p-1) !\left|\epsilon_{1}\right|<m|\bar{\alpha}| e^{|\bar{\alpha}|}|\bar{\alpha}|^{p-1} H^{p},
$$

where $|\bar{a}|$ stands for the maximum $\left|\alpha_{i}\right|$, and where

$$
H=\max _{|z| \leq|\bar{\alpha}|}\left|\Pi\left(z-\alpha_{i}\right) \ldots \Pi\left(z-\sigma_{i}\right)\right||(A B \cdots S)|^{d} .
$$

The factor $m$ in (25) arises from the summation in (23), the first $|\bar{\alpha}|$ arises from the path of integration, and the remaining factors from the integrand. Similarly for $\epsilon_{2}, \cdots, \epsilon_{n}$. To complete the proof pick $p>G$ so that $M=\bar{m}(p)$, then pick $p>a_{0}$ so that $a_{0} M=\bar{m}(p)$, and finally pick $p$ so large that the part of (4) involving the $\epsilon$ 's is less than 1 in absolute value.

5. Algebraic part of the proof. Having proved Theorem 1, we show that the Lindemann theorem can be deduced from it. The proof involves three stages, described by Lemmas 9,11 , and 12 ; the final result is stated as Theorem 2.

LEMMA 9. If e satisfies an equation of the type considered in the Lindemann theorem, then $e$ satisfies an equation of the same type in which $a, b, \cdots, s$ are integers.

To establish Lemma 9, consider the expression $a z^{\alpha}+b z^{\beta}+\cdots+s z^{\sigma}$ which arises from the expression in the Lindemann theorem when we replace $e$ by a variable $z$. Since at least one of the numbers $a, b, \ldots, s$ is different from zero we may assume that they all are. Suppose $a$ has conjugates $a_{1}=a, a_{2}, \ldots, a_{m}$, while $b$ has conjugates $b_{j}, \cdots$, and $s$ has conjugates $s_{k}$. Form the product of the various expressions that arise when $a, b, \ldots, s$ are replaced by their conjugates, thus:

$$
\begin{gathered}
\prod_{i, j, \cdots, k}\left(a_{i} z^{\alpha}+b_{j} z^{\beta}+\cdots+s_{k} z^{\sigma}\right) \\
\equiv A z^{\rho_{1}}+B z^{\rho_{2}}+\cdots+K z^{\rho_{n}} .
\end{gathered}
$$

It is understood that all formally distinct factors are present in (26); the number of factors is equal to the product of degrees of the irreducible equations satisfied by $a, b, \ldots, s$. In (27) we have collected terms and simplified as much as possible, so that no two exponents $\rho_{k}$ are equal. This is important.

At least one coefficient in (27) is different from zero. For, if not, the product (26) is identically zero; by Lemma 7 one of the factors in (26) is identically zero; and by Lemma 6, then, each of the coefficients in this factor is zero. But the original coefficients $a, b, \ldots, s$ are nonzero by hypothesis, and the conjugates are nonzero by definition. 
We assert next that the coefficients $A, B, \ldots, K$ in (27) are rational. For every $z$, it is clear that the product $(26)$ is a symmetric function in the $a_{i}$ 's, regarded as variables; interchanging two $a$ 's only changes the order of the factors in (26). By Lemma 8 , the coefficients $A, B, \ldots, K$ in (27) are also symmetric functions of the $a$ 's. Similarly, they are symmetric in the $b$ 's, $\cdots$, and in the $s$ 's. Since (27) is obtained by expanding (26), the coefficients $A, B, \cdots, K$ are polynomials in the $a_{i}, b_{j}, \cdots, s_{k}$ with integral coefficients. By Lemma 2 , then, they are rational.

When $z$ has the value $e$, the expression (26) vanishes, since the first factor does. Therefore (27) also vanishes when $z=e$, and Lemma 9 follows when we multiply (27) by a suitably chosen integer.

LEMMA 10. If $e$ satisfies an equation of the type considered in the conclusion of Lemma 9, then e satisfies an equation of the form

$$
a e^{a_{1}}+b e^{a_{2}}+\cdots+s e^{a_{n}}=0 \text {, }
$$

where $a, b, \ldots, s$ are integers not all zero, where the exponents $a_{i}$ are all of the roots of a polynomial equation with integral coefficients, and where no two of these exponents are equal.

The proof of Lemma 10 involves little more than a change in notation. In the conclusion of Lemma 9 we may assume that all the coefficients $a, b, \ldots, s$ are integers different from zero. It may happen that some of the conjugates of $\alpha$ appear among the other exponents $\beta, \cdots, \sigma$. Collect these together at the beginning of the expression, and add enough terms, taking zero coefficients, so that not only some, but all of the conjugates of $\alpha$ appear. Thus one obtains

$$
\left(a_{1} e^{a_{1}}+a_{2} e^{\alpha_{2}}+\cdots+a_{n} e^{a_{n}}\right)+g e^{\gamma}+\cdots+f e^{\phi}=0
$$

where $\alpha_{1}=\alpha, \alpha_{2}, \ldots, \alpha_{n}$ are the conjugates of $\alpha$, and where $\gamma, \ldots, \phi$ are a subset of the exponents $\beta, \cdots, \sigma$ in (1). Repeat, starting with the term $e^{\gamma}$, and so on.

We note, first, that the process terminates after a finite number of steps, since there are only finitely many exponents in (1). Second, no two exponents in the final result are equal, by Lemma 4 . Third, at least one coefficient is different from zero; an example is $a_{1}=a$. Finally, the exponents are the roots of the product of the various irreducible polynomials which have $\alpha_{i}, \gamma_{j}, \ldots$ as their roots; and since these polynomials have integral coefficients, so does their product. Lemma 10 now follows by a trivial change in notation.

LEMMA 11. If e satisfies an equation of the type considered in the con- 
clusion of Lemma 10, then e satisfies an equation of the type (18), where the $\alpha$ 's are all the roots of an irreducible equation with integral coefficients, the $\beta$ 's are all the roots of a different equation of the same type, and so on, up to the $\sigma$ 's, which are the roots of still another such equation. The value of $a_{0}$, however, may be zero.

Starting from the result mentioned in the conclusion of Lemma 10, we take the product of all expressions that can be obtained by permuting the $\alpha$ 's. This product is to be computed formally, without any simplification or combining of terms; the formal character may be emphasized by considering the $a$ 's and $\alpha$ 's as variables. Each term of the product has the form $R e^{Q}$, where $R$ is a product of the $a$ 's, and $Q$ is a linear combination of the $\alpha$ 's with integral coefficients. Symbolically,

$$
\begin{gathered}
\prod\left(a_{1} e^{\alpha_{i}}+a_{2} e^{\alpha_{j}}+\cdots+a_{n} e^{a_{k}}\right) \quad(i, j, \cdots, k \neq) \\
\equiv \sum R_{k} e^{Q_{k}}
\end{gathered}
$$

with $n$ ! factors in (28), $n^{n !}$ terms in (29).

If we change the order of the factors in (28), we get exactly the same product, hence the same collection of terms (29). Now, interchanging two of the $\alpha$ 's amounts merely to changing the order of the factors in (28); hence such an interchange does not alter the collection (29). (It is insufficient for our purposes that the value of the sum (29) is unaltered; we need to know that the sums obtained before and after interchange actually consist of the same terms. That is why we speak of a "collection" rather than a "sum").

Let us fix attention on a particular product of the $a$ 's, say $R_{p}$, and collect all terms in the sum (29) that contain $R_{p}$ as factcr:

$$
R_{p}\left(e^{Q_{m}}+e^{Q_{k}}+\cdots+e^{Q_{i}}\right) .
$$

By the previous remarks the collection of terms (30) remains the same after any interchange of the $\alpha$ 's, and hence the exponents in (30) are merely permuted by such an interchange. Thus it is that the product

$$
f(t)=\prod\left(t-Q_{j}\right),
$$

where $Q_{j}$ runs over the set of exponents in (30), is a symmetric function of the $\alpha$ 's: interchanging two $\alpha$ 's permutes the $Q$ 's in (30), hence permutes the factors in (31), hence does not alter $f(t)$. By Lemmas 8 and 2, then, $f(t)$ has rational coefficients. 
Lemma 3 shows that $f(t)$ in (31) can be written as a product of irreducible polynomials over the rationals. Corresponding to each factor of $f(t)$ is a set of $Q$ 's, which we lump together to form a block of terms of the type desired in Lemma 11. This process is repeated for each (different) $R$ in (29), until the $R$ 's are used up. Finally, we group together any blocks of terms that may be identical, to obtain a sum of the type described in the conclusion of Lemma 11.

We must still show that at least one coefficient is different from zero. But this follows at once as in the paragraph after (27): replace $e$ by a variable $z$, recall that the $\alpha$ 's in (28) are unequal by Lemma 10, and use Lemmas 7 and 6 .

We remark in passing that the work up to this point already contains the fact that $\pi$ is transcendental [3, (cf. also [6]). For, if $i \pi$ is an algebraic number $\alpha$, we have $1+e^{\alpha}=0$, and the product over the conjugates gives

$$
0=\prod\left[1+e^{\alpha_{i}}\right]=1+e^{\beta_{1}}+e^{\beta_{2}}+\cdots+e^{\beta_{n}} .
$$

The terms for which $\beta_{k}=0$ combine with the 1 to give a term $a_{0} \geq 1$, and a simplified version of the discussion leading to Lenima 11 shows that the remaining $\beta$ 's are all the roots of a polynomial equation with integral coefficients. Theorem 1 now applies.

LEMMA 12. If $e$ satisfies an equation of the type considered in the conclusion of Lemma 11, then e satisfies an equation of the type (18).

The equation reached in the conclusion of Lemma 11 has the form (18) with $a_{0}$ possibly equal to zero and with the $\alpha$ 's all the roots of one irreducible equation, the $\beta$ 's of another, and so on. The coefficients $a, b, \ldots$ are not zero, by Lemma 11, and no two exponents are equal, in view of Lemma 4 . Our task is to produce a nonzero term $a_{0}$ if it is not already present.

To this end, we suppose $a_{0}$ absent and multiply (18) by $e^{-\sigma_{1}}+e^{-\sigma_{2}}+\cdots+$ $e^{-\sigma_{j}}$. Actual calculation gives

$$
\left(e^{-\sigma_{1}}+e^{-\sigma_{2}}+\cdots+e^{-\sigma_{j}}\right)\left(e^{\alpha_{1}}+e^{\alpha_{2}}+\cdots+e^{\alpha_{m}}\right)=\sum_{i, j} e^{-\sigma_{i}+\alpha_{j}} .
$$

The exponents on the right of (32) are the roots of $\Pi\left(t-\left(\alpha_{j}-\sigma_{i}\right)\right]=0$, which has rational coefficients by the (now familiar) use of Lemmas 8 and 2 . Since none of these exponents is zero-this is important - we get a block of terms like those in (18). Similarly for $\left(\sum e^{-\sigma_{i}}\right)\left(\sum e^{\beta_{j}}\right)$, and so on. But when we reach the $\sigma$ 's the situation changes: now some of the exponents are zero. Specifically, we have

$$
\left(\sum e^{-\sigma_{i}}\right)\left(\sum e^{\sigma_{j}}\right)=\sum e^{0}+\sum_{i \neq j} e^{-\sigma_{i}+\sigma_{j}}
$$


where the terms $e^{0}$ arise from the choice $i=j$. These furnish the term $a_{0}$ that we are seeking: $a_{0}=m s$. As to the other terms in (33), the exponents are nonzero, and are the roots of

$$
\prod_{i \neq j}\left[t-\left(\sigma_{j}-\sigma_{i}\right)\right]=0
$$

Thus we have proved:

THEOREM 2. If $e$ satisfies an equation of the type considered in the Lindemann theorem, then e satisfies an equation of the type considered in Theorem 1.

\section{REFERENCES}

1. G. Birkhoff and S. MacLane, A survey of modern algebra, Macmillan, 1941.

2. C. Hermite, Sur la fonction exponentielle, C. R. Acad. Sci., Paris 77 (1873), 18-24.

3. D. Hilbert, Ueber die Transcendenz der Zahlen e und $\pi$, Math. Ann. 43 (1893), 216-219.

4. F. Klein, Famous problems of elementary geometry, Ginn and Co., 1897.

5. F. Lindemann, Ueber die Zahl $\pi$, Math. Ann., 20 (1882), 213-225.

6. I. Niven, A simple proof that $\pi$ is irrational, Bull. Amer. Math. Soc. 53 (1947), 509.

7. C. L. Siegel, Transcendental numbers, Ann. of Math. Studies No. 16, 1949, Princeton Univ. Press. This reference contains a bibliography.

8. T. Skolem, A proof of the algebraic independence of certain values of the exponential function. Norske Vid. Selsk. Forhandlinger, 19, No. 12, p. 40.

9. J. V. Uspensky, Theory of equations, McGraw-Hill (1948), p.63, p. 264.

10. K. Weierstrass, $Z u$ Lindemann's Adhandlung, "Über die Ludolph'sche Zahl", Math. Werke II, (1895), 341-362. 


\section{PACIFIC JOURNAL OF MATHEMATICS}

\section{EDITORS}

R. M. RoBINSON

University of California Berkeley 4, California

\section{*R. P. Dilworth}

Califomia Institute of Technology Pasadena 4, California

\section{E. F. BECKENBACH, Managing Editor}

University of California

Los Angeles 24, California

*During the absence of Herbert Busemann in 1952.

\section{ASSOCIATE EDITORS}

\author{
R. P. DILWORTH \\ HERBERT FEDERER \\ MARSHALL HALL
}

\author{
P. R. HALMOS \\ HEINZ HOPF
}

R. D. JAMES

\author{
BØRGE JESSEN \\ PAUL LÉVY \\ GEORGE PÓLYA
}

\author{
J. J. STOKER \\ E. G. STRAUS
}

KÖSAKU YOSIDA

\section{SPONSORS}

UNIVERSITY OF BRITISH COLUMBIA

CALIFORNIA INSTITUTE OF TECHNOLOGY

UNIVERSITY OF CALIFORNIA, BERKELEY

UNIVERSITY OF CALIFORNIA, DAVIS

UNIVERSITY OF CALIFORNIA, LOS ANGELES

UNIVERSITY OF CALIFORNIA, SANTA BARBARA

OREGON STATE COLLEGE

UNIVERSITY OF OREGON

\author{
UNIVERSITY OF SOUTHERN CALIFORNIA \\ STANFORD UNIVERSITY \\ WASHINGTON STATE COLLEGE \\ UNIVERSITY OF WASHINGTON \\ AMERICAN MATHEMATICAL SOCIETY \\ NATIONAL BUREAU OF STANDARDS, \\ INSTITUTE FOR NUMERICAL ANALYSIS
}

Mathematical papers intended for publication in the Pacific Journal of Miathematics should be typewritten (double spaced), and the author should keep a complete copy. Manuscripts may be sent to any of the editors. All other communications to the editors should be addressed to the managing editor, E. F. Beckenbach, at the address given above.

Authors are entitled to receive 100 free reprints of their published papers and may obtain additional copies at cost.

The Pacific Journal of Mathematics is published quarterly, in March, June, September, and December, by the University of California, Berkeley 4, California. The price per volume (4 numbers) is $\$ 8.00$; single issues, $\$ 2.50$. Special price to individual faculty members of supporting institutions and to individual members of the American Mathematical Society: $\$ 4.00$ per volume; single issues, $\$ 1.25$.

Subscriptions, orders for back numbers, and changes of address should be sent to the publishers, University of California Press, Berkeley 4, California.

Printed at Ann Arbor, Michigan. Entered as second class matter at the Post Office, Berkeley, California.

\section{UNIVERSITY OF CALIFORNIA PRESS • BERKELEY AND LOS ANGELES}




\section{Pacific Journal of Mathematics}

\section{Vol. 2, No. $2 \quad$ February, 1952}

L. Carlitz, Some theorems on Bernoulli numbers of higher order...

Watson Bryan Fulks, On the boundary values of solutions of the heat equation........................................ 141

John W. Green, On the level surfaces of potentials of masses with fixed center of gravity................................... 147

Isidore Heller, Contributions to the theory of divergent series .......... 153

Melvin Henriksen, On the ideal structure of the ring of entire functions . . . 179

James Richard Jackson, Some theorems concerning absolute neighborhood retracts........................................ 185

Everett H. Larguier, Homology bases with applications to local connectedness ................................... 191

Janet McDonald, Davis's canonical pencils of lines ................ 209

J. D. Niblett, Some hypergeometric identities . . . . . . . . . . . . . . . . . . 219

Elmer Edwin Osborne, On matrices having the same characteristic equation...................................... 227

Robert Steinberg and Raymond Moos Redheffer, Analytic proof of the

Lindemann theorem ................................. 231

Edward Silverman, Set functions associated with Lebesgue area ......... 243

James G. Wendel, Left centralizers and isomorphisms of group algebras . . 251

Kosaku Yosida, On Brownian motion in a homogeneous Riemannian space. 